PROCEEDINGS OF THE

AMERICAN MATHEMATICAL SOCIETY

Volume 140, Number 4, April 2012, Pages 1185-1197

S 0002-9939(2011)10979-1

Article electronically published on July 29, 2011

\title{
CANONICAL CURVES ON SURFACES OF VERY LOW DEGREE
}

\author{
G. CASNATI
}

(Communicated by Lev Borisov)

\begin{abstract}
Let $C$ be a non-hyperelliptic curve of genus $g$. We recall some facts about curves endowed with a base-point-free $g_{4}^{1}$. Then we prove that if the minimal degree of a surface containing the canonical model of $C$ in $\check{\mathbb{P}}_{k}^{g-1}$ is $g$, then $7 \leq g \leq 12$ and $C$ carries exactly one $g_{4}^{1}$. As a by-product, we deduce that if the canonical model of $C$ in $\check{\mathbb{P}}_{k}^{g-1}$ is contained in a surface of degree at most $g$, then $C$ is either trigonal or tetragonal or isomorphic to a plane sextic.
\end{abstract}

\section{INTRODUCTION AND NOTATION}

Let $k$ be an algebraically closed field and denote by $\operatorname{char}(k)$ its characteristic. Let $C$ be a curve (i.e. a smooth, connected, projective scheme of dimension 1) and denote by $g \geq 2$ its genus. The canonical system on $C$ gives rise to a natural morphism $\phi: C \rightarrow \check{\mathbb{P}}_{k}^{g-1}$. The map $\phi$ is either the double cover of a rational normal curve or it is an isomorphism. In the former case $C$ is said to be hyperelliptic and in the latter $C_{c a n}:=\phi(C)$ is called a canonical model of $C$.

The study of the intrinsic properties of a non-hyperelliptic curve $C$ is closely related to the description of the embedding $C_{c a n} \subseteq \check{\mathbb{P}}_{k}^{g-1}$.

For example, one could inspect some properties of surfaces $S \subseteq \check{\mathbb{P}}_{k}^{g-1}$ (i.e. integral, projective schemes of dimension 2) containing $C_{c a n}$. Notice that such surfaces must be obviously non-degenerate, i.e. not contained in any hyperplane of $\check{\mathbb{P}}_{k}^{g-1}$. Such a viewpoint has been adopted in $\mathrm{C}-\mathrm{H}$. The authors prove that, if $C$ is general in its moduli space and its genus is $g \geq 23$, then $\operatorname{deg}(S) \geq 2 g-3$. They also characterise the surfaces for which such a minimum value is attained.

A second viewpoint consists in understanding whether some particular geometric characteristics of surfaces $S \supseteq C_{c a n}$ reflect important properties of $C$. For example, one could try to classify curves $C$ for which $d:=\operatorname{deg}(S)$ is very low. Due to the geometric characterisation of the degree, we know that $\operatorname{deg}(S) \geq g-2$. It is thus natural to ask what happens when $d=g-2, g-1, g$, and so on. A first natural step in this direction could consist of understanding the nature of such surfaces of very low degree.

Received by the editors October 14, 2010 and, in revised form, December 15, 2010; December 26, 2010; and December 29, 2010.

2010 Mathematics Subject Classification. Primary 14N25; Secondary 14H51, 14H30, 14N05.

Key words and phrases. Curve, canonical model, tetragonality, Maroni number, apolarity.

This work was done in the framework of PRIN 'Geometria delle varieté a algebriche e dei loro spazi di moduli', cofinanced by MIUR (COFIN 2008).

(C)2011 American Mathematical Society Reverts to public domain 28 years from publication 
The classification of surfaces of minimal degree (i.e with $d=g-2$ ) is due to del Pezzo (see dP1; see also Fj3 and $[\mathrm{E}-\mathrm{H}]$ ). Also thanks to such a classification, one obtains the Babbage-Enriques-Noether-Petri Theorem (see [A-C-G-H] p. 131, when $\operatorname{char}(k)=0$ and [SD] when $\operatorname{char}(k)>0)$.

Theorem A. Let $C$ be a non-hyperelliptic curve of genus $g \geq 3$. Then $C_{\text {can }} \subseteq \check{\mathbb{P}}_{k}^{g-1}$ is contained in an integral surface $S$ of degree $g-2$ if and only if exactly one of the two following conditions holds:

i) $C$ is trigonal (i.e. there exists a map $C \rightarrow \mathbb{P}_{k}^{1}$ which is finite of degree 3 ): in this case $S$ is a rational normal scroll.

ii) $g=6$ and $C$ is isomorphic to a plane quintic: in this case $S$ is a Veronese surface.

Also the classification of surfaces of almost minimal degree (i.e. with $d=g-1$ ) is known (see $\mathrm{dP} 2$; see also $\mathrm{Fj} 1, \mathrm{Fj} 2, \mathrm{Fj} 3, \mathrm{H}-\mathrm{W},[\mathrm{Bd}-\mathrm{Sc}$ ). The following result has been proven in $[\mathrm{B}-\mathrm{C}-\mathrm{N}]$.

Theorem B. Let $C$ be a non-hyperelliptic curve of genus $g \geq 5$. Then $C_{\text {can }} \subseteq \check{\mathbb{P}}_{k}^{g-1}$ is contained in an integral surface $S$ of degree $g-1$ if and only if one of the three following conditions holds:

i) $C$ is bielliptic (i.e. there exists a double cover $C \rightarrow E$ onto an elliptic curve): in this case $S$ is a cone on an elliptic normal curve contained in a hyperplane of $\check{\mathbb{P}}_{k}^{g-1}$ with vertex not on $C_{\text {can }}$.

ii) $g \leq 10$ and $C$ is birationally isomorphic to a plane sextic carrying $10-g$, possibly infinitely near, double points: in this case $S$ is the anticanonical image of the blow up of $\mathbb{P}_{k}^{2}$ at such points.

iii) $g=9$ and $C$ is isomorphic to a smooth complete intersection inside $\mathbb{P}_{k}^{3}$ of an integral quadric $Q$ with an integral quartic surface: in this case $S$ is the anticanonical embedding of $Q$.

The main result of this paper is the answer to the following question: What can be said if the canonical model of $C$ in $\check{\mathbb{P}}_{k}^{g-1}$ is contained in an integral surface $S$ of degree $g$ ? We succeed in completely answering this question in Section 4.

Theorem C. Let $C$ be a non-hyperelliptic curve of genus $g$. If the minimal degree of a surface $S \subseteq \check{\mathbb{P}}_{k}^{g-1}$ containing $C_{\text {can }}$ is $g$, then $7 \leq g \leq 12$ and $S$ is a conic bundle over $\mathbb{P}_{k}^{1}$ whose fibres cut out on $C$ a base-point-free $g_{4}^{1}$. The curve $C$ does not carry any other base-point-free $g_{4}^{1}$.

The quickest way to achieve such a result does not pass through any kind of classification of surfaces $S \subseteq \check{\mathbb{P}}_{k}^{g-1}$ of degree $g$. It suffices to combine the description of the residual linear system cut out on $S$ by the quadrics through $C_{c a n}$ with some well-known facts about base-point-free $g_{4}^{1}$ 's on a curve $C$.

We recall such results in Sections 2 and 3, proving Theorem C in Section 4. We also prove therein some corollaries and check that the above result is sharp, at least when $k$ is the complex field. Finally, in Section 5, we show how Theorem $\mathrm{C}$ above is related to an invariant of a non-hyperelliptic curve, namely its apolarity, introduced for the first time in $[\mathrm{I}-\mathrm{R}]$ (see also $[\mathrm{R}-\mathrm{S}]$ ) and investigated in some recent papers (see e.g. [DP-Z1], [DP-Z2] and [B-C-N]).

Notation. We work over an algebraically closed field $k$. We denote by $\operatorname{char}(k)$ its characteristic. 
If $V$ is a vector space, then we denote by $\mathbb{P}(V)$ the corresponding projective space. In particular, we set $\mathbb{P}_{k}^{n}:=\mathbb{P}\left(k^{n+1}\right)$. A curve in Section 2 is an integral and projective scheme of dimension 1, without embedded components. From Section 3, a curve is also assumed to be smooth.

Let $X \subseteq \mathbb{P}_{k}^{n}$ be a closed subscheme and let $\mathcal{L}$ be a sheaf on $X$. If $q \in H^{0}(X, \mathcal{L})$ we will denote by $D_{0}(q) \subseteq X$ its zero-scheme.

Let $\gamma:=\left(\gamma_{0}, \ldots, \gamma_{n}\right) \in \mathbb{N}^{n+1}$ be a multi-index. We set $|\gamma|:=\sum_{i=0}^{n} \gamma_{i}, \gamma !:=$ $\prod_{i=0}^{n} \gamma_{i} !$, and $t^{\gamma}:=t_{0}^{\gamma_{0}} \ldots t_{n}^{\gamma_{n}} \in k\left[t_{0}, \ldots, t_{n}\right]$. We say that $\gamma \geq 0$ if and only if $\gamma_{i} \geq 0$ for each $i=0, \ldots, n$. If $\delta:=\left(\delta_{0}, \ldots, \delta_{n}\right) \in \mathbb{N}^{n+1}$ is another multi-index, then we write $\gamma \geq \delta$ if and only if $\gamma-\delta \geq 0$.

For other definitions, results and notation we always refer to $\mathrm{Ha}$.

\section{Covers of Degree 4}

We start by recalling some standard facts about covers of degree 4 . If $C$ is a curve, then a morphism $\varrho: C \rightarrow \mathbb{P}_{k}^{1}$ is a cover of degree 4 if it is finite, flat and $\operatorname{rk}\left(\varrho_{*} \mathcal{O}_{C}\right)=4$. In this case there exists a natural exact sequence

$$
0 \longrightarrow \mathcal{O}_{\mathbb{P}_{k}^{1}} \stackrel{\varrho^{\#}}{\longrightarrow} \varrho_{*} \mathcal{O}_{C} \longrightarrow \check{\mathcal{E}} \longrightarrow 0
$$

where $\check{\mathcal{E}}$ is a locally free $\mathcal{O}_{\mathbb{P}_{k}^{1}}$-sheaf of rank 3 called the Tschirnhausen module of $\varrho$.

Since every locally free $\mathcal{O}_{\mathbb{P}_{k}^{1}}$-sheaf splits, we can fix a decomposition $\mathcal{E} \cong \bigoplus_{i=1}^{3} \mathcal{O}_{\mathbb{P}_{k}^{1}}\left(\alpha_{i}\right)$. Sequence (2.1) and Serre duality yields $g+3=\alpha_{1}+\alpha_{2}+\alpha_{3}$.

Theorem 2.2. Let $C$ be a curve of genus $g$ and $\varrho: C \rightarrow \mathbb{P}_{k}^{1}$ a cover of degree 4. There exists an embedding $i: C \hookrightarrow \mathbb{P}:=\mathbb{P}(\mathcal{E}) \stackrel{\pi}{\longrightarrow} \mathbb{P}_{k}^{1}$, a locally free $\mathcal{O}_{\mathbb{P}_{k}^{1}}$-sheaf $\mathcal{F}:=\bigoplus_{j=1}^{2} \mathcal{O}_{\mathbb{P}_{k}^{1}}\left(\beta_{j}\right)$ such that $\sum_{j=1}^{2} \beta_{j}=g+3$ and a morphism $\delta: \pi^{*} \mathcal{F} \stackrel{\delta^{k}}{\longrightarrow} \mathcal{O}_{\mathbb{P}}(2)$ such that $C=D_{0}(\delta)$ and $\varrho=\pi_{\mid D_{0}}(\delta)$. Moreover,

$$
\omega_{C} \cong i^{*}\left(\mathcal{O}_{\mathbb{P}}(1) \otimes \pi^{*} \mathcal{O}_{\mathbb{P}_{k}^{1}}(-2)\right)
$$

Conversely let $\mathcal{E} \cong \bigoplus_{i=1}^{3} \mathcal{O}_{\mathbb{P}_{k}^{1}}\left(\alpha_{i}\right)$ and $\mathcal{F} \cong \bigoplus_{j=1}^{2} \mathcal{O}_{\mathbb{P}_{k}^{1}}\left(\beta_{j}\right)$ be such that $\sum_{i=1}^{3} \alpha_{i}=$ $\sum_{j=1}^{2} \beta_{j}$, define $\mathbb{P}:=\mathbb{P}(\mathcal{E}) \stackrel{\pi}{\longrightarrow} \mathbb{P}_{k}^{1}$ and let $\delta: \pi^{*} \mathcal{F} \rightarrow \mathcal{O}_{\mathbb{P}}(2)$. If $\varrho=\pi_{\mid D_{0}(\delta)}$ is a cover, then it has degree 4 and the Tschirnhausen module of $\varrho$ is $\check{\mathcal{E}}$.

Proof. See [C-E], Theorem 2.1 and Section 4.

Definition 2.3. Let $C$ be a curve, $\varrho: C \rightarrow \mathbb{P}_{k}^{1}$ a cover of degree 4 and fix decompositions $\mathcal{E} \cong \bigoplus_{i=1}^{3} \mathcal{O}_{\mathbb{P}_{k}^{1}}\left(\alpha_{i}\right), \alpha_{1} \leq \alpha_{2} \leq \alpha_{3}, \mathcal{F} \cong \bigoplus_{j=1}^{2} \mathcal{O}_{\mathbb{P}_{k}^{1}}\left(\beta_{j}\right), \beta_{1} \leq \beta_{2}$, of the associated sheaves.

The triple $\alpha:=\left(\alpha_{1}, \alpha_{2}, \alpha_{3}\right)$ is called the scrollar invariant of $\varrho$. The number $\tau:=\beta_{1}-4$ is called the tetragonality of $\varrho$.

Both the scrollar invariants and the tetragonality are classically well-known invariants associated to each $g_{4}^{1}$ on a curve.

We have three monomorphisms $\mathcal{O}_{\mathbb{P}_{k}^{1}}\left(\alpha_{i}\right) \longmapsto \mathcal{E}$ into the three summands, hence three fibrewise independent sections $u \in H^{0}\left(\mathbb{P}, \mathcal{O}_{\mathbb{P}}(1) \otimes \pi^{*} \mathcal{O}_{\mathbb{P}_{k}^{1}}\left(-\alpha_{1}\right)\right) \cong$ $H^{0}\left(\mathbb{P}_{k}^{1}, \mathcal{E}\left(-\alpha_{1}\right)\right), v \in H^{0}\left(\mathbb{P}, \mathcal{O}_{\mathbb{P}}(1) \otimes \pi^{*} \mathcal{O}_{\mathbb{P}_{k}^{1}}\left(-\alpha_{2}\right)\right) \cong H^{0}\left(\mathbb{P}_{k}^{1}, \mathcal{E}\left(-\alpha_{2}\right)\right)$, and $w \in$ $H^{0}\left(\mathbb{P}, \mathcal{O}_{\mathbb{P}}(1) \otimes \pi^{*} \mathcal{O}_{\mathbb{P}_{k}^{1}}\left(-\alpha_{3}\right)\right) \cong H^{0}\left(\mathbb{P}_{k}^{1}, \mathcal{E}\left(-\alpha_{3}\right)\right)$.

Via the isomorphism

$H^{0}\left(\mathbb{P}, \mathcal{O}_{\mathbb{P}}(2) \otimes \pi^{*} \check{\mathcal{F}}\right) \cong H^{0}\left(\mathbb{P}, \mathcal{O}_{\mathbb{P}}(2) \otimes \pi^{*} \mathcal{O}_{\mathbb{P}_{k}^{1}}\left(-\beta_{1}\right)\right) \oplus H^{0}\left(\mathbb{P}, \mathcal{O}_{\mathbb{P}}(2) \otimes \pi^{*} \mathcal{O}_{\mathbb{P}_{k}^{1}}\left(-\beta_{2}\right)\right)$, 
we can identify $\delta$ with a pair $(a, b)$ where

$$
\begin{aligned}
a(u, v, w)=a_{2 \alpha_{1}-\beta_{1}} u^{2} & +a_{\alpha_{1}+\alpha_{2}-\beta_{1}} u v+a_{\alpha_{1}+\alpha_{3}-\beta_{1}} u w \\
& +a_{2 \alpha_{2}-\beta_{1}} v^{2}+a_{\alpha_{2}+\alpha_{3}-\beta_{1}} v w+a_{2 \alpha_{3}-\beta_{1}} w^{2}, \\
b(u, v, w)=b_{2 \alpha_{1}-\beta_{2}} u^{2} & +b_{\alpha_{1}+\alpha_{2}-\beta_{2}} u v+b_{\alpha_{1}+\alpha_{3}-\beta_{2}} u w \\
& +b_{2 \alpha_{2}-\beta_{2}} v^{2}+b_{\alpha_{2}+\alpha_{3}-\beta_{2}} v w+b_{2 \alpha_{3}-\beta_{2}} w^{2},
\end{aligned}
$$

$a_{h}, \quad b_{h} \in H^{0}\left(\mathbb{P}_{k}^{1}, \mathcal{O}_{\mathbb{P}_{k}^{1}}(h)\right)$. In particular, let $A:=D_{0}(a), B:=D_{0}(b)$. Then $C=A \cap B \subseteq \mathbb{P}$. Since both $C$ and $\mathbb{P}$ are smooth, it follows that the surfaces $A$ and $B$ are smooth at the points of $C$.

Proposition 2.5. Let $C$ be a curve of genus $g \geq 4, \varrho: C \rightarrow \mathbb{P}_{k}^{1}$ a cover of degree $4, \alpha:=\left(\alpha_{1}, \alpha_{2}, \alpha_{3}\right)$ and $\tau$ the corresponding invariants. Then

$$
\frac{g+3}{3} \geq \alpha_{1} \geq 1, \quad \min \left\{\frac{g-5}{2}, 2\left(\alpha_{1}-2\right)\right\} \geq \tau \geq-2 .
$$

Proof. By definition, $\alpha_{1} \leq \alpha_{2} \leq \alpha_{3}$ and $\beta_{1} \leq \beta_{2}$. Thus the inequalities

$$
\alpha_{1} \leq \frac{g+3}{3}, \quad \tau \leq \frac{g-5}{2}
$$

are immediate. Since $h^{0}\left(\mathbb{P}_{k}^{1}, \mathcal{O}_{\mathbb{P}_{k}^{1}}\right)=h^{0}\left(C, \mathcal{O}_{C}\right)=1$ and $h^{1}\left(\mathbb{P}_{k}^{1}, \mathcal{O}_{\mathbb{P}_{k}^{1}}\right)=0$, taking the cohomology of sequence $(2.1)$, one also easily deduces that $h^{0}\left(\mathbb{P}_{k}^{1}, \check{\mathcal{E}}\right)=0$, whence $\alpha_{1} \geq 1$.

If $2\left(\alpha_{1}-2\right)<\tau$, then in equations (2.4) above we would have $a_{2 \alpha_{1}-\beta_{1}}=$ $b_{2 \alpha_{1}-\beta_{2}}=0$. Hence the unisecant curve $D_{0}(v, w) \subseteq \mathbb{P}$ would be contained in $C$, contradicting the irreducibility of $C$.

If $\tau<-2$, then $\beta_{1} \leq 1$ and $\beta_{2} \geq g+2$. If $\alpha_{1} \leq \alpha_{2} \leq \alpha_{3}<(g+2) / 2$, then $b_{\alpha_{i}+\alpha_{j}-\beta_{2}}=0$ for each $i, j=1,2,3$ in equations (2.4). Thus $b(u, v, w)=0$; hence $C=A$, a contradiction. It follows that $\alpha_{3} \geq(g+2) / 2$. Since $\alpha_{1} \geq 1$, we deduce that $\alpha_{2} \leq(g+2) / 2$. If $\alpha_{1}>1$, then $b(u, v, w)=b^{\prime} v w+b^{\prime \prime} w^{2}$, where $b^{\prime}, b^{\prime \prime}$ are forms of non-negative degree.

Thus $B$ splits in $\mathbb{P}$ as the union of the two subbundles $W:=D_{0}(w)$ and $B^{\prime}:=$ $D_{0}\left(b^{\prime} v+b^{\prime \prime} w\right)$. In particular, $C=C_{1} \cup C_{2}$, where $C_{1}:=C \cap W$ and $C_{2}:=C \cap B^{\prime}$. Since $C$ is irreducible, it follows that either $C=C_{1}$ or $C=C_{2}$. In any case we obtain a contradiction, since $C$ is fibrewise the complete intersection of two conics.

We deduce that $\alpha_{1}=1, \alpha_{2}=\alpha_{3}=(g+2) / 2$. If $g \geq 1$, then $b(u, v, w)=$ $b^{\prime} v^{2}+b^{\prime \prime} v w+b^{\prime \prime \prime} w^{2}$, where $b^{\prime}, b^{\prime \prime}, b^{\prime \prime \prime}$ are constants. Thus $B$ would again be reducible, and an argument analogous to the one used above yields a contradiction. We conclude that the bound $\beta_{1} \geq 2$ must hold, whence $\tau \geq-2$.

\section{Tetragonality And CANONICAL EMBEDDing}

For the rest of the paper the word curve will always denote a smooth, integral, projective scheme of dimension 1 . Since we are interested in the surfaces of the canonical space containing $C_{c a n}$, we restrict our attention to the case $g \geq 4$.

Recall that a curve $C$ of genus $g \geq 2$ is called hyperelliptic if there exists a cover $\varphi: C \rightarrow \mathbb{P}_{k}^{1}$ of degree 2 . Every hyperelliptic curve $C$ carries infinitely many $g_{4}^{1}$ 's obtained by composing $\varphi$ with any double cover $\mathbb{P}_{k}^{1} \rightarrow \mathbb{P}_{k}^{1}$. In this section we will first study some different characterisations of base-point-free $g_{4}^{1}$ 's on a 
non-hyperelliptic curve $C$ of genus $g \geq 2$ in terms of its scrollar invariant $\alpha$ and tetragonality $\tau$. We start recalling the following well-known results.

Proposition 3.1. Let $C$ be a non-hyperelliptic curve of genus $g \geq 4$ and $\varrho: C \rightarrow \mathbb{P}_{k}^{1}$ a cover of degree $4, \alpha:=\left(\alpha_{1}, \alpha_{2}, \alpha_{3}\right)$ and $\tau$ the corresponding invariants. Then the following assertions hold:

i) $\alpha_{1} \geq 2$ and $\tau \geq-1$.

ii) If $\tau<(g-9) / 3$, then $\tau=2\left(\alpha_{1}-2\right)$.

iii) If $\tau=-1$, then $C$ is either trigonal or isomorphic to a smooth plane quintic. If this happens, then $g \leq 6$.

Proof. In order to prove assertion i) it suffices to check that $\tau \geq-1$, due to Proposition 2.5. However, this has been proven in Section 6.3 of [Sch1, taking into account that the number $b_{2}$ defined there is exactly $\tau=\beta_{1}-4$.

For the proof of assertion ii) see [Sch2], Corollary 4.2. For the first part of the proof of assertions iii) see Section (6.3) and (6.4) of [Sch1. The bound on the genus follows from assertion ii), since $\tau=-1$ is odd.

Now let $C$ be a non-hyperelliptic curve of genus $g \geq 4$. Let $\varrho: C \rightarrow \mathbb{P}_{k}^{1}$ be a cover of degree 4 and consider the corresponding invariants $\alpha:=\left(\alpha_{1}, \alpha_{2}, \alpha_{3}\right)$ and $\tau$. Due to Proposition $3.1 \mathrm{i}$ ), one deduces that $\alpha_{1} \geq 2$ and $\tau \geq-1$. As in the previous section we set $\mathbb{P}:=\mathbb{P}(\mathcal{E})$.

The Picard group $\operatorname{Pic}(\mathbb{P})$ of $\mathbb{P}$ is generated by the class $\xi$ of the tautological sheaf $\mathcal{O}_{\mathbb{P}}(1)$ and by the class $f$ of a fibre of $\pi$. Moreover, the Chern equation on $\mathbb{P}$ becomes

$$
\xi^{3}=(g+3) \xi^{2} f
$$

The linear system $|\xi-2 f|$ on $\mathbb{P}$ is globally generated (since $\alpha_{1} \geq 2$ ) and it defines a morphism $\phi: \mathbb{P} \rightarrow \Sigma:=\operatorname{im}(\phi) \subseteq \check{\mathbb{P}}_{k}^{g-1}$ onto a rational normal scroll. The restriction of $\phi$ to $C$ is exactly the canonical embedding (see formula (2.2.1)). If $\alpha_{1}>2$, then $\phi$ is actually an embedding. Notice that this happens as soon as $\tau \geq 1$, thanks to Proposition 2.5. If $\alpha_{1}=2$, then $\phi$ is no longer an embedding. In this case (see [Sch1], Section 1.1 and the references therein) $\Sigma$ is singular and $\phi$ is a resolution of its singularities.

In any case the planes in $\mathbb{P}$ cutting the $g_{4}^{1}$ on $C$ are mapped on planes in $\Sigma$; in particular, the divisor on the $g_{4}^{1}$ are cut on $C_{c a n}$ by planes.

The curve $C$ is the complete intersection inside $\mathbb{P}$ of the two conic bundles $A \in$ $|2 \xi-(\tau+4) f|$ and $B \in|2 \xi-(g-1-\tau) f|$. Thus $C_{c a n} \subseteq \check{\mathbb{P}}_{k}^{g-1}$ coincides with the intersection of the two surfaces $A_{c a n}:=\phi(A)$ and $B_{c a n}:=\phi(B)$ whose degrees are

$$
\begin{gathered}
\operatorname{deg}\left(A_{\text {can }}\right)=(2 \xi-(\tau+4) f)(\xi-2 f)^{2}=2 g-6-\tau, \\
\operatorname{deg}\left(B_{\text {can }}\right)=(2 \xi-(g-1-\tau) f)(\xi-2 f)^{2}=g-1+\tau .
\end{gathered}
$$

Since $\tau \leq(g-5) / 2$, it follows that $\operatorname{deg}\left(B_{\text {can }}\right) \leq \operatorname{deg}\left(A_{\text {can }}\right)$. We can summarise the above remarks in the following proposition.

Proposition 3.2. Let $C$ be a non-hyperelliptic curve of genus $g \geq 4, \varrho: C \rightarrow \mathbb{P}_{k}^{1}$ a cover of degree 4 with tetragonality $\tau$. Then the $g_{4}^{1}$ on $C$ corresponding to $\varrho$ is cut out on $C_{\text {can }}$ by a family of planes in $\mathbb{P}_{k}^{g-1}$ whose union $\Sigma$ is a scroll over $\mathbb{P}_{k}^{1}$. Moreover, $C_{\text {can }}$ is the complete intersection inside $\Sigma$ of two conic bundles $A_{\text {can }}$ and $B_{\text {can }}$ of respective degrees $2 g-6-\tau$ and $g-1+\tau$. If $\tau \geq 1$, then $\Sigma$ is smooth. 
One can be even more precise. Indeed, we have the following nice geometric characterisation of the tetragonality of a cover (for a more precise result obtained via different methods, see $[\mathrm{Bn}-\mathrm{Sa}]$ ).

Theorem 3.3. Let $C$ be a non-hyperelliptic curve of genus $g \geq 4, \varrho: C \rightarrow \mathbb{P}_{k}^{1}$ a cover of degree 4 with tetragonality $\tau$ and let $\Sigma \subseteq \check{\mathbb{P}}_{k}^{g-1}$ be as above. Then $2 g-6-\tau$ is the minimal degree of an integral surface $S \subseteq \Sigma$, different from $B_{\text {can }}$ and containing $C_{c a n}$.

Proof. Let $\alpha:=\left(\alpha_{1}, \alpha_{2}, \alpha_{3}\right)$ and $\tau$ be the invariants associated to $\varrho$. Since $C$ is non-hyperelliptic, we have that $\tau \geq-1$ and $\alpha_{1} \geq 2$ (see Proposition $3.1 \mathrm{i}$ )).

If $g=4$, then $\alpha=(2,2,3)$ and $\beta=(3,4)$, whence $\tau=-1$. In this case, $\Sigma=\check{\mathbb{P}}_{k}^{g-1}$, and it is well known that $C$ is the complete intersection inside $\check{\mathbb{P}}_{k}^{g-1}$ of a surface of degree 3 with a quadric which has degree $2=g-1+\tau$.

Now, we assume $g \geq 5$. Let $\lambda \xi-\mu f$ be the class of $\phi^{-1}(S) \subseteq \mathbb{P}$ in $\operatorname{Pic}(\mathbb{P})$ : trivially we have that $\lambda \geq 2$. If we assume that

$$
\operatorname{deg}(S)=(\lambda \xi-\mu f)(\xi-2 f)^{2}=\lambda(g-1)-\mu<2 g-6-\tau,
$$

then we have

$$
-\mu<(2-\lambda)(g-1)-\tau-4 .
$$

If $S \neq B_{\text {can }}$, then $S \cap B_{\text {can }}$ is a curve of degree

$\operatorname{deg}\left(S \cap B_{\text {can }}\right)=(2 \xi-(g-1-\tau) f)(\lambda \xi-\mu f)(\xi-2 f)=\lambda(g-1)-2 \mu+(\tau+4) \lambda$.

Inequality (3.3.1) thus yields

$$
\operatorname{deg}\left(S \cap B_{\text {can }}\right)<2 g-2-(\lambda-2)(g-5-\tau) .
$$

On the one hand $\tau \leq(g-5) / 2$ and $g \geq 5$, whence $g-5-\tau \geq 0$ for each $\tau \geq-1$. On the other hand $\lambda-2 \geq 0$ since $C_{c a n} \subseteq S$. We conclude that

$$
2 g-2=\operatorname{deg}\left(C_{c a n}\right) \leq \operatorname{deg}\left(S \cap B_{\text {can }}\right)<2 g-2-(\lambda-2)(g-5-\tau) \leq 2 g-2,
$$

and this is a contradiction.

We conclude this section with the following lemma for which we are unable to give a correct reference. Recall that a curve $C$ is called tetragonal if and only if it carries a $g_{4}^{1}$ but it does not carry any $g_{d}^{1}$ with $d \leq 3$.

Lemma 3.4. Let $C$ be a tetragonal curve of genus $g \geq 5$. If $\varrho_{1}: C \rightarrow \mathbb{P}_{k}^{1}$ and $\varrho_{2}: C \rightarrow \mathbb{P}_{k}^{1}$ are distinct covers of degree 4 with tetragonalities $\tau_{1}$ and $\tau_{2}$ respectively, then $\tau_{1}=\tau_{2}=0$. If $C$ is not bielliptic, then $g \leq 9$.

Proof. For the proof of the equalities $\tau_{1}=\tau_{2}=0$, see Sch1, Section 6.3. We have a well-defined morphism $\psi=\varrho_{1} \times \varrho_{2}: C \rightarrow \mathbb{P}_{k}^{1} \times \mathbb{P}_{k}^{1}$ and $\psi(C)$ is a divisor of bidegree $(4,4)$ on $\mathbb{P}_{k}^{1} \times \mathbb{P}_{k}^{1}$. If $\psi$ is birational, then the genus of $C$ is at most 9 .

If $\psi$ is not birational, then it is generically finite onto its reduced image $C_{0}:=$ $\operatorname{im}(\psi)_{\text {red }}$. The degree $\operatorname{deg}(\psi)$ of $\psi$ is at most 3 , since $\varrho_{1} \neq \varrho_{2}$. It follows that $\operatorname{deg}(\psi)=2$ necessarily, thus $C_{0}$ is a curve of bidegree $(2,2)$. We deduce that $C_{0}$ is either rational or it is a smooth elliptic curve. The first case cannot occur; otherwise $C$ would be hyperelliptic. It follows that it must be bielliptic. 
Let $C$ be tetragonal. In particular, we are implicitly assuming that $g \geq 5$ and that $C$ is neither hyperelliptic nor trigonal. It follows from Proposition 3.1 that the tetragonality $\tau$ of the corresponding cover $\varrho: C \rightarrow \mathbb{P}_{k}^{1}$ is at least 0 . Lemma 3.4 allows us to introduce the following definition.

Definition 3.5. Let $C$ be a tetragonal curve. The tetragonality $\tau(C)$ of $C$ is the common value of the tetragonalities of all the covers $\varrho: C \rightarrow \mathbb{P}_{k}^{1}$ of degree 4 .

\section{Proof of Theorem C}

In this section we prove Theorem $\mathrm{C}$ stated in the introduction. We will also check that Theorem $\mathrm{C}$ is sharp, at least when $\operatorname{char}(k)=0$.

Proof of Theorem $C$. The minimal degree of a surface containing $C_{c a n}$ is $g$. Then $C$ is neither trigonal nor tetragonal with $\tau=0$ (by Theorems A and B); thus $g \geq 7$ and $C_{c a n}$ is cut out by quadrics. Each quadric through $C_{c a n}$ cut out on $S$, residually to $C_{\text {can }}$, a curve $\Gamma$ such that $\operatorname{deg}(\Gamma)=2 \operatorname{deg}(S)-\operatorname{deg}\left(C_{c a n}\right)=2$. If $C_{\text {can }} \subseteq \operatorname{Sing}(S)$, then $2=\operatorname{deg}(\Gamma) \geq \operatorname{deg}\left(C_{c a n}\right)=2 g-2$, a contradiction. Thus $C_{c a n} \not \subset \operatorname{Sing}(S)$.

Let us consider a minimal desingularization $p: \widetilde{S} \rightarrow S$ (see ZZ when $\operatorname{char}(k)=0$ and $\mathrm{Ab}$ when $\operatorname{char}(k)>0$ ). Let $\widetilde{C}$ be the strict transform of $C_{\text {can }}$ in $\widetilde{S}$ and denote by $H$ the total transform of a general hyperplane section of $S$. The pull-back of the linear system of quadrics through $C_{c a n}$ has a fixed part $F$, which is mapped by $p$ to the singular points of $S$, and a non-empty movable part $|M|$ : we have $H \cdot M=\operatorname{deg}(\Gamma)=2$. We can thus write $2 H \sim \widetilde{C}+M+F$, whence

$$
2 H \cdot M=\widetilde{C} \cdot M+M^{2}+F \cdot M,
$$

where $\widetilde{C} \cdot M, M^{2}, F \cdot M$ are all non-negative. Since $2 g-2=\widetilde{C} \cdot H>M \cdot H=2$, it follows that $h^{0}\left(\widetilde{S}, \mathcal{O}_{\widetilde{S}}(M-\widetilde{C})\right)=0$. Thus the cohomology of the exact sequence

$$
0 \longrightarrow \mathcal{O}_{\widetilde{S}}(M-\widetilde{C}) \longrightarrow \mathcal{O}_{\widetilde{S}}(M) \longrightarrow \mathcal{O}_{\widetilde{C}}(M) \longrightarrow 0
$$

yields

$$
h^{0}\left(\widetilde{C}, \mathcal{O}_{\widetilde{C}}(M)\right) \geq h^{0}\left(\widetilde{S}, \mathcal{O}_{\widetilde{S}}(M)\right) \geq 2 .
$$

If either $M^{2} \geq 1$ or $F \cdot M \geq 1$, then $\widetilde{C} \cdot M \leq 3$. Thanks to inequality (4.1) it follows the existence of a base-point-free $g_{d}^{1}$ on $C \cong \widetilde{C}$ with $d \leq 3$, a contradiction, since $C$ is neither hyperelliptic nor trigonal.

We conclude that $\widetilde{C} \cdot M=4, M^{2}=F \cdot M=0$. Several facts follow from these equalities. First, a unique element of $|M|$ passes through each point of $\widetilde{S}$. Second, $C$ carries a $g_{4}^{r}$ with $r \geq 1$; thus $C \cong \widetilde{C}$ is tetragonal (recall that $C$ is neither hyperelliptic nor trigonal). Moreover, if $r \geq 2$, the curve $C$ would be hyperelliptic, since $g \geq 4$. Thus $|M|$ cut out a complete $g_{4}^{1}$, and it follows that inequalities (4.1) are actually equalities, whence $|M|^{\vee} \cong \mathbb{P}_{k}^{1}$. Let $\varrho: C \rightarrow \mathbb{P}_{k}^{1}$ be the tetragonal map induced by $|M|$ and let $\Sigma \subseteq \check{\mathbb{P}}_{k}^{g-1}$ be the corresponding scroll (see Proposition 3.2).

Assume that the general element of $|M|$ is not reduced. Since $M \cdot H=2$, for such a general element we necessarily have $M=2 M_{\text {red }}$ in $\operatorname{Pic}(\widetilde{S})\left(M_{\text {red }}\right.$ is the reduced scheme structure on $M$ ) and $M_{\text {red }} \cdot H=1$. Since $M \cdot \widetilde{C}=4$, it follows that $M_{\text {red }} \cdot \widetilde{C}=2$. Recall that a unique element of $|M|$ passes through each point of $\widetilde{S}$. Thus we would obtain a natural rational map $\widetilde{S} \rightarrow|M|^{2} \cong \mathbb{P}_{k}^{1}$. Such a map induces by restriction a well-defined rational map $\widetilde{C} \rightarrow \mathbb{P}_{k}^{1}$, which actually extends 
to a morphism, since $\widetilde{C}$ is smooth. Moreover the degree of such a morphism is 2 since $M_{\text {red }} \cdot \widetilde{C}=2$. We deduce that $C$ should be hyperelliptic, a contradiction. We conclude that the general element in $|M|$ is necessarily reduced.

Let $\Gamma$ be the image of a general element of $|M|$. It follows that $\Gamma$ is a reduced curve of degree 2 , thus it is either a conic or it is the union of two skew lines. On the one hand, by construction, $\Gamma$ cuts on $C_{c a n}$ the general element of the aforementioned $g_{4}^{1}$. On the other hand, it follows from Proposition 3.2 that each element of the $g_{4}^{1}$ is the complete intersection of a suitable pencil of conics in a plane: such a complete intersection cannot be contained in the union of two skew lines. We conclude that $\Gamma$ is a conic in a plane cutting the general element of the $g_{4}^{1}$ on $C_{\text {can }}$. Since $\Sigma$ is exactly the union of such kind of planes, we also have that it contains $\Gamma$. It follows that $S \subseteq \Sigma$, thus $g \geq g-1+\tau$ (see Theorem 3.3), i.e. $\tau \leq 1$. If the strict equality holds, then $C_{c a n}$ would be contained in a surface of degree at most $g-1$, contradicting the minimality of $\operatorname{deg}(S)$. We conclude that $\tau=1$, which is not even, so that $g \leq 12$ by Proposition 3.1 ii). Since $1=\tau \leq(g-5) / 2$ (see Proposition 2.5), we also have that $g \geq 7$.

Finally, if $C$ were endowed with two different covers $\varrho_{i}: C \rightarrow \mathbb{P}_{k}^{1}, i=1,2$, then $\tau_{1}, \tau_{2}=0$ (Lemma 3.4). Thus $C_{c a n}$ would be contained in the surface $B_{c a n}$, which has degree $g-1<g$ by Theorem 3.3. This last fact contradicts the hypothesis. We conclude that $C$ carries exactly one $g_{4}^{1}$.

The following corollaries to Theorems A, B, C are immediate.

Corollary 4.2. Let $C$ be a non-hyperelliptic curve of genus $g$. If $C_{c a n}$ is contained in a surface $S \subseteq \check{\mathbb{P}}_{k}^{g-1}$ of degree $g$, then $C$ is either trigonal or tetragonal or isomorphic to a plane sextic.

Corollary 4.3. Let $C$ be a non-hyperelliptic, non-trigonal curve of genus $g \geq 13$. If $C_{\text {can }}$ is contained in a surface $S \subseteq \check{\mathbb{P}}_{k}^{g-1}$ of degree $g$, then $C$ is bielliptic.

We conclude this section by proving that Theorem $\mathrm{C}$ is sharp when $\operatorname{char}(k)=0$; i.e. for each $g=7, \ldots, 12$ there always exists a curve $C$ of genus $g$ such that the minimal degree of a surface $S \subseteq \check{\mathbb{P}}_{k}^{g-1}$ containing $C_{\text {can }}$ is $g$.

Due to Theorem $\mathrm{C}$ all such curves are necessarily endowed with a cover $\varrho: C \rightarrow$ $\mathbb{P}_{k}^{1}$ of degree 4 . Looking at the proof of Theorem $\mathrm{C}$, we know that we have to ask for those covers such that $\tau=3$.

The following Bertini-type theorem will be used.

Theorem 4.4. Let $\mathcal{E}:=\bigoplus_{i=1}^{3} \mathcal{O}_{\mathbb{P}_{k}^{1}}\left(\alpha_{i}\right)$ and $\mathcal{F}:=\bigoplus_{j=1}^{2} \mathcal{O}_{\mathbb{P}_{k}^{1}}\left(\beta_{j}\right)$ be such that $\sum_{i=1}^{3} \alpha_{i}=\sum_{j=1}^{2} \beta_{j}$. Define $\mathbb{P}:=\mathbb{P}(\mathcal{E}) \stackrel{\pi}{\longrightarrow} \mathbb{P}_{k}^{1}$ and

$$
\begin{aligned}
\mathcal{U}:=\{(a, b) \in & H^{0}\left(\mathbb{P}, \mathcal{O}_{\mathbb{P}}(2) \otimes \pi^{*} \check{\mathcal{F}}\right) \mid \\
& \left.D_{0}(a, b) \text { is smooth and } \pi_{\mid D_{0}(a, b)} \text { is a cover of degree } 4\right\} .
\end{aligned}
$$

Then $\mathcal{U}$ is open. If $\operatorname{char}(k)=0$ and $S^{2} \mathcal{E} \otimes \check{\mathcal{F}}$ is globally generated, then $\mathcal{U} \neq \emptyset$.

Proof. See [C-E], Theorem 4.5, and [CS, Theorem 2.5.

Proposition 4.5. Let $\operatorname{char}(k)=0$. For each $g=7, \ldots, 12$ there exists a nonhyperelliptic curve $C$ of genus $g$ such that the minimal degree of a surface $S \subseteq \check{\mathbb{P}}_{k}^{g-1}$ containing $C_{c a n}$ is $g$. 
Proof. For each $\alpha:=\left(\alpha_{1}, \alpha_{2}, \alpha_{3}\right)$ and $\beta:=\left(\beta_{1}, \beta_{2}\right)$ we define $\mathcal{E}_{\alpha}:=\bigoplus_{i=1}^{3} \mathcal{O}_{\mathbb{P}_{k}^{1}}\left(\alpha_{i}\right)$ and $\mathcal{F}_{\beta}:=\bigoplus_{j=1}^{2} \mathcal{O}_{\mathbb{P}_{k}^{1}}\left(\beta_{j}\right)$.

For $g \neq 11$ we choose $\alpha$ and $\beta$ satisfying $\sum_{i=1}^{3} \alpha_{i}=\sum_{j=1}^{2} \beta_{j}=g+3$ in such a way that $S^{2} \mathcal{E}_{\alpha} \otimes \check{\mathcal{F}}_{\beta}$ is globally generated. Thus, for a general choice of $(a, b) \in$ $H^{0}\left(\mathbb{P}, \mathcal{O}_{\mathbb{P}}(2) \otimes \pi^{*} \check{\mathcal{F}}\right)$, the subscheme $D_{0}(a, b)$ is a smooth curve and $\pi_{\mid D_{0}(a, b)}$ is a cover of degree 4 (due to Theorem 5.4) of genus $g$ (due to Theorem 2.2).

If $g=12$ we set $\alpha:=(5,5,5)$ and $\beta:=(5,10)$. If $g=10$ we set $\alpha:=(4,4,5)$ and $\beta:=(5,8)$. If $g=9$ we set $\alpha:=(4,4,4)$ and $\beta:=(5,7)$. If $g=8$ we set $\alpha:=(3,4,4)$ and $\beta:=(5,6)$. If $g=7$ we set $\alpha:=(3,3,4)$ and $\beta:=(5,5)$.

Now we examine separately the case $g=11$. Notice that in this case we cannot argue as above, so we will construct directly a cover $\varrho: C \rightarrow \mathbb{P}_{k}^{1}$ with $\tau=3$

Choose a point $p \in \mathbb{P}_{k}^{1} \times \mathbb{P}_{k}^{1}$ and denote by $\mathbb{F}$ the blow up of $\mathbb{P}_{k}^{1} \times \mathbb{P}_{k}^{1}$ at $p$. Then $\operatorname{Pic}(\mathbb{F})$ is freely generated by the classes of the total transforms $\eta$ and $h$ of the two general members of the pencils of lines on $\mathbb{P}_{k}^{1} \times \mathbb{P}_{k}^{1}$ and by the class $e$ of the exceptional divisor.

Consider a smooth and integral divisor $C \in|4 \eta+5 h-2 e|$ : the curve $C$ has degree 11 and it is endowed with a base-point-free $g_{4}^{1}$ cut out by $|h|$. One checks that the linear system $\Gamma:=|2 \eta+3 h-e|$ on $\mathbb{F}$ is base-point-free, very ample on an open subset of $\mathbb{F}$ and it maps birationally $\mathbb{F}$ in $\check{\mathbb{P}}_{k}^{g-1}$ as a conic bundle $B$ of degree 11 . The restriction $\Gamma_{\mid C}$ is the canonical system on $C$ which is thus non-hyperelliptic. Finally $B$ is contained in the 3 -dimensional scroll $\Sigma$ generated by the $g_{4}^{1}$ on $C$; thus $\tau \leq 1$. If the strict inequality holds, then $\operatorname{deg}(B)$ should be at least 16 , by Theorem 3.3. We conclude that $\tau=1$.

\section{Apolarity of a tetragonal Curve}

We start this section by recalling some facts about the classical Macaulay correspondence. We refer to $[\mathrm{I}-\mathrm{K}]$ (see also $[\mathrm{R}-\mathrm{S}]$ and $[\mathrm{I}-\mathrm{R}]$ for the characteristic 0 case) for other details and the proofs.

We recall that we can consider two graded rings, namely the polynomial ring $T^{n}:=k\left[x_{0}, \ldots, x_{n}\right]$ and the divided power $k$-algebra $R^{n}$ in the $n+1$ variables $y_{0}, \ldots, y_{n}$.

As explained in $[\mathrm{I}-\mathrm{K}]$, Appendix $\mathrm{A}$, the $k$-vector space $R^{n}$ coincides with $k\left[y_{0}, \ldots, y_{n}\right]$; thus there exists a natural action of $\mathrm{GL}_{n+1}$ on $R^{n}$. Let $\gamma:=\left(\gamma_{0}, \ldots, \gamma_{n}\right)$ $\in \mathbb{N}^{n+1}$ : the monomial $y_{0}^{\gamma_{0}} \ldots y_{n}^{\gamma_{n}} \in R^{n}$ is usually denoted by $y^{[\gamma]}$. The $k$-algebra structure on $R^{n}$ is obtained by extending by linearity the monomial multiplication

$$
y^{[\gamma]} y^{[\delta]}:=\frac{(\gamma+\delta) !}{\gamma ! \delta !} y^{[\gamma+\delta]} .
$$

The algebra $T^{n}$ acts on $R^{n}$ by differentiation. More precisely, if $\gamma:=\left(\gamma_{0}, \ldots, \gamma_{n}\right)$ and $\delta:=\left(\delta_{0}, \ldots, \delta_{n}\right) \in \mathbb{N}^{n+1}$ we have the natural pairing $T_{g}^{n} \times R_{d}^{n} \rightarrow R_{d-g}^{n}$ (we denote by $*_{d}^{n}$ the summand of degree $d$ elements in $*^{n}$ ) given on monomials by the rule

$$
x^{\gamma}\left(y^{[\delta]}\right):= \begin{cases}y^{[\delta-\gamma]} & \text { if } \delta \geq \gamma, \\ 0 & \text { if } \delta \geq \gamma .\end{cases}
$$


For any linear form $\ell:=\sum_{i=0}^{n} a_{i} y_{i} \in R_{1}^{n}$ the divided power $\ell^{[d]}$ is defined as

$$
\ell^{[d]}:=\sum_{\delta \in \mathbb{N}^{n+1},|\delta|=d} a_{0}^{\delta_{0}} \ldots a_{n}^{\delta_{n}} y_{0}^{\left[\delta_{0}\right]} \ldots y_{n}^{\left[\delta_{n}\right]} .
$$

Such an action defines a perfect pairing $T_{d}^{n} \times R_{d}^{n} \rightarrow k$ between forms of degree $d$ in $R^{n}$ and in $T^{n}$. In particular, $R_{1}^{n}$ and $T_{1}^{n}$ are natural dual vector spaces. Therefore the projective spaces with coordinates $y_{0}, \ldots, y_{n}$ and $x_{0}, \ldots, x_{n}$ are naturally dual to each other, and we denote them by $\mathbb{P}_{k}^{n}$ and $\check{\mathbb{P}}_{k}^{g-1}$ respectively.

Two homogeneous forms $f \in R^{n}$ and $q \in T^{n}$ are called apolar if $q(f)=0$. As explained in [I-K], apolarity allows us to associate an Artinian Gorenstein graded quotient of $T^{n}$ to a form in $R^{n}$ as follows. If $f \in R_{d}^{n}$, then

$$
f^{\perp}:=\left\{q \in T^{n} \mid q(f)=0\right\}
$$

is easily seen to be a homogeneous ideal in $T^{n}$ and $A^{f}:=T^{n} / f^{\perp}$ is an Artinian Gorenstein graded quotient of $T^{n}$ with socle $0:_{T^{n}} T_{1}^{n}$ in degree $d$. Also, the converse holds: if $A$ is an Artinian Gorenstein graded quotient of $T^{n}$, say $A:=T^{n} / I$, with socle in degree $d$, then there exists $f \in R_{d}^{n}$ such that $I=f^{\perp}$. The main result about apolarity, due to Macaulay (see [I-K], Lemma 2.12 and the references therein), is the following theorem.

Theorem 5.1. The map $f \mapsto A^{f}$ induces a bijection between $\mathbb{P}\left(R_{d}^{n}\right)$ and the set of graded Artinian Gorenstein quotient rings of $T^{n}$ with socle in degree $d$.

Now we restrict our attention to non-hyperelliptic curves $C$ of genus $g \geq 3$. Then the homogeneous coordinate ring of $C_{c a n}$ inside $\check{\mathbb{P}}_{k}^{g-1}$ satisfies

$$
T_{C}:=\bigoplus_{h=0}^{+\infty} H^{0}\left(C, \omega_{C}^{h}\right)
$$

If we take a general linear subspace $H=D_{0}\left(h_{1}, h_{2}\right) \subseteq \check{\mathbb{P}}_{k}^{g-1}$ of codimension 2, then the algebra $T_{C} /\left(h_{1}, h_{2}\right)$ turns out to be naturally an Artinian Gorenstein graded quotient of $T^{g-1} /\left(h_{1}, h_{2}\right)$ with socle in degree 3 and Hilbert function $(1, g-$ $2, g-2,1)$. With a proper choice of coordinates $y_{0}, \ldots, y_{g-1}$ in $\mathbb{P}_{k}^{g-1}$ and of the corresponding dual coordinates $x_{0}, \ldots, x_{g-1}$ in $\check{\mathbb{P}}_{k}^{g-1}$, we can assume that $H=$ $V\left(x_{g-2}, x_{g-1}\right)$ so that $T^{g-1} /\left(h_{1}, h_{2}\right) \cong T^{g-3}$.

Thus we can associate to $C$ and $H$ a polynomial $f \in R_{3}^{g-3}$ (up to the natural action of $\left.\mathrm{GL}_{g-3}\right)$ in such a way that

$$
T_{C} /\left(x_{g-2}, x_{g-1}\right) \cong T^{g-3} / f^{\perp} .
$$

Definition 5.2. With the notation introduced above we say that the polynomial $f \in R^{g-3}$ and the curve $C$ are apolar to each other.

Following the definition given in $\underline{I-R}$ in the case $\operatorname{char}(k)=0$, apolarity allows us to define a rational map

$$
\psi_{C}: G(g-2, g) \rightarrow H_{g-3,3},
$$

where $G(g-2, g)$ denotes the Grassmannian of subspaces of codimension 2 of $\check{\mathbb{P}}_{k}^{g-1}$ and $H_{g-3,3}:=R_{3}^{g-3} / \mathrm{GL}_{g-2}$, without restrictions on $\operatorname{char}(k)$. On the other hand, in $H_{g-3,3}$ is also defined the locus $H_{g-3,3}(h)$ of $\mathrm{GL}_{g-2}$-orbits of cubics (in the sense of a divided power algebra when $\operatorname{char}(k)>0$ ) which can be written as a sum of $h$ (divided power) cubes of linear forms. Obviously we must have $h \geq 1$ and 
$H_{g-3,3}(h) \subseteq H_{g-3,3}(h+1)$. For simplicity we denote by ap $(C)$ the apolarity of $C$, i.e. the smallest integer $h$ such that the general point of $\operatorname{im}\left(\psi_{C}\right)$ is in $H_{g-3,3}(h)$.

A natural problem is to inspect curves $C$ with low apolarity. To this purpose a fundamental tool is the following fundamental result, known as the Apolarity Lemma.

Lemma 5.3. Let $\ell_{i} \in R_{1}^{n}$ and let $L_{i}:=D_{0}\left(\ell_{i}\right) \subseteq \check{\mathbb{P}}_{k}^{g-1}, i=1, \ldots, s$. Then $f=\sum_{i=0}^{s} \lambda_{i} \ell_{i}^{[d]}$ for some $\lambda_{i} \in k^{*}, i=0, \ldots, s$, if and only if the homogeneous ideal $I_{\Gamma} \subseteq R^{n}$ of $\Gamma:=\left\{L_{0}, \ldots, L_{s}\right\} \subset \check{\mathbb{P}}_{k}^{g-1}$ satisfies $I_{\Gamma} \subseteq f^{\perp}$.

Proof. See $[\mathrm{B}-\mathrm{C}-\mathrm{N}]$, Lemma 1.6, and the references therein.

The condition $I_{\Gamma} \subseteq f^{\perp}$ is often summarised in the standard literature by saying that $\Gamma$ is apolar to $f$.

By combining the above result with Theorems A and B stated in the introduction and Theorems 1.7 and 4.2 of [B-C-N] (see also Theorem 4 of [DP-Z1]), one can prove the following.

Theorem 5.4. Let $C$ be a non-hyperelliptic curve $C$ of genus $g \geq 5$. The minimal degree of a surface $S \subseteq \check{\mathbb{P}}_{k}^{g-1}$ containing $C_{\text {can }}$ is $g-2$ (resp. $g-1$ ) if and only if $\operatorname{ap}(C)=g-2($ resp. ap $(C)=g-1)$.

When $C$ is tetragonal we are able to prove the following partial result.

Proposition 5.5. Let $C$ be a tetragonal curve of genus $g \geq 5$. Then

$$
\operatorname{ap}(C) \leq g-1+\tau(C) \leq g-1+\left\lfloor\frac{g-5}{2}\right\rfloor .
$$

Proof. Recall that for a tetragonal curve of genus $g \geq 5$ one has $\tau \geq 0$; hence $g-1+\tau \geq g-1$.

Let $C=A \cap B$ be the corresponding representation in $\mathbb{P}$ with the standard meaning of symbols. Let $H:=D_{0}\left(h_{1}, h_{2}\right) \subseteq \check{\mathbb{P}}_{k}^{g-1}$, where $h_{1}$ and $h_{2}$ are general linear forms. The geometric characterisation of the degree of a surface allows us to assume that $\Gamma:=B_{c a n} \cap H$ consists of exactly $g-1+\tau$ pairwise distinct points.

We know that $I_{\Gamma}$ is the saturation of $I_{B_{c a n}}+\left(h_{1}, h_{2}\right)$. By construction we have

$$
I_{B_{\text {can }}}+\left(h_{1}, h_{2}\right) \subseteq I_{C_{c a n}}+\left(h_{1}, h_{2}\right) .
$$

The ideal on the right is saturated, since the same is true for $I_{C_{c a n}}$ and $C_{c a n}$ is arithmetically Gorenstein (see Mi, Proposition 1.3.4). Thus, by saturating both sides of the above inclusion, the ideal on the right does not change. Modulo $\left(h_{1}, h_{2}\right)$, we finally obtain

$$
I_{\Gamma \mid H} \subseteq \frac{I_{C_{c a n}}+\left(h_{1}, h_{2}\right)}{\left(h_{1}, h_{2}\right)} .
$$

We can assume that $h_{i}=x_{g-3+i}, i=1,2$, so that $I_{\Gamma \mid H} \subseteq k\left[x_{0}, \ldots, x_{g-3}\right]$ and

$$
\frac{T(C)}{\left(h_{1}, h_{2}\right)} \cong \frac{k\left[x_{0}, \ldots, x_{g-3}\right]}{f^{\perp}} \text {. }
$$

Thus $I_{\Gamma \mid H} \subseteq f^{\perp}$. It then follows from Lemma 5.3 that the general point of $\operatorname{im}\left(\psi_{C}\right)$ is in $H_{g-3,3}(g-1+\tau)$; hence $\operatorname{ap}(C) \leq g-1+\tau$.

It is thus natural, at this point, to ask the following question. 
Question 5.6. Let $g \geq 5$ and let $C$ be a tetragonal curve. Is it true that $\operatorname{ap}(C)=$ $g-1+\tau(C)$ ?

An immediate consequence of Theorem C, Theorem 5.4 and Proposition 5.5 is the following proposition.

Proposition 5.7. Let $C$ be a non-hyperelliptic curve of genus $g \geq 3$. If the minimal degree of a surface $S \subseteq \check{\mathbb{P}}_{k}^{g-1}$ containing $C_{\text {can }}$ is $g$, then $\operatorname{ap}(C)=g$.

Proof. On the one hand, due to Theorem $\mathrm{C}$, we have $g \geq 7, C$ is tetragonal and $\tau(C)=1$. Proposition 5.5 thus implies that ap $(C) \leq g-1+\tau(C)=g$. On the other hand, due to Theorem 5.4 we have that ap $(C) \geq g$. We conclude that $\operatorname{ap}(C)=g$.

\section{REFERENCES}

[Ab] S. S. Abhyankar, Local uniformization on algebraic surfaces over ground fields of characteristic $p \neq 0$, Ann. of Math. (2), 63 (1956), 491-526. MR0078017 (17:1134d)

[A-C-G-H] E. Arbarello, M. Cornalba, P. A. Griffiths, J. Harris, Geometry of algebraic curves, Vol. I, Springer-Verlag, 1985. MR0770932 (86h:14019)

[B-C-N] E. Ballico, G. Casnati, R. Notari, Canonical curves with low apolarity, J. Algebra, 332 (2011), 229-243.

[Bd-Sc] M. Brodmann, P. Schenzel, Arithmetic properties of projective varieties of almost minimal degree, J. Algebraic Geom., 16 (2007), 347-400. MR.2274517|(2008b:14085)

[Bn-Sa] M. Brundu, G. Sacchiero, Stratification of the moduli space of four-gonal curves, preprint.

[Cs] G. Casnati, Covers of algebraic varieties III. The discriminant of a cover of degree 4 and the trigonal construction, Trans. Amer. Math. Soc., 350 (1998), 1359-1378. MR.1467462 (98i:14021)

[C-E] G. Casnati, T. Ekedahl, Covers of algebraic varieties I. A general structure theorem, covers of degree 3, 4 and Enriques surfaces, J. Algebraic Geom., 5 (1996), 439-460. MR.1382731 (97c:14014)

[C-H] C. Ciliberto, J. Harris, Surfaces of low degree containing a general canonical curve, Comm. Algebra, 27 (1999), 1127-1140. MR.1669124 (2000c:14051)

[dP1] P. del Pezzo, Sulle superficie di ordine $n$ nello spazio di $n+1$ dimensioni, Rend. R. Acc. delle Scienze Fisiche e Mat. di Napoli, 24 (1885), 212-216.

[dP2] P. del Pezzo, Sulle superficie di ordine $n$ nello spazio di $n$ dimensioni, Rend. del Circolo Mat. di Palermo, 1 (1886), 241-271.

[DP-Z1] P. De Poi, F. Zucconi, Gonality, apolarity, and hypercubics, J. London Math. Soc., to appear.

[DP-Z2] P. De Poi, F. Zucconi, Fermat hypersurfaces and subcanonical curves, arXiv:0908.0522.

[E-H] D. Eisenbud, J. Harris, On varieties of minimal degree (a centennial account), Algebraic Geometry, Bowdoin, 1985, Spencer J. Bloch, Proceedings of Symposia in Pure Mathematics, 46, 3-13, AMS, 1987. MR927946 (89f:14042)

[Fj1] T. Fujita, Classification of projective varieties of $\Delta$-genus one, Proc. Japan Acad. - Ser. A, 58 (1982), 113-116. MR664549 (83g:14003)

[Fj2] T. Fujita, Projective varieties of $\Delta$-genus one, Algebraic and topological theories. Papers from the symposium dedicated to the memory of Dr. Takehiko Miyata (Kinokuniya, Tokyo, 1985), M. Nagata, S. Araki, A. Hattori, N. Iwahori et al. (eds.), Kinokuniya Company Ltd., 1986, 149-175. MR1102257

[Fj3] T. Fujita, Classification theories of polarized varieties, London Mathematical Society Lecture Notes Series, 155, Cambridge University Press, 1990. MR1162108 (93e:14009)

[Ha] R. Hartshorne, Algebraic geometry, Springer, 1977. MR0463157 (57:3116)

$[\mathrm{H}-\mathrm{W}] \quad$ F. Hidaka, K. Watanabe, Normal Gorenstein surfaces with ample anti-canonical divisor, Tokyo J. Math., 4 (1981), 319-330. MR646042 (83h:14031) 
[I-K] A. Iarrobino, V. Kanev, Power sums, Gorenstein algebras, and determinantal loci, Lecture Notes in Mathematics, 1721, Springer, 1999. MR.1735271 (2001d:14056)

[I-R] A. Iliev, K. Ranestad, Canonical curves and varieties of sums of powers of cubic polynomials, J. Algebra, 246 (2001), 385-393. MR.1872627(2003c:14032)

[Mi] J. C. Migliore, Introduction to liaison theory and deficiency modules, Progress in Mathematics, 165, Birkhäuser, 1998. MR.1712469 (2000g:14058)

[R-S] K. Ranestad, F. O. Schreyer, Varieties of sums of powers, J. Reine Angew. Math., $\mathbf{5 2 5}$ (2000), 147-181. MR1780430 (2001m:14009)

[SD] B. Saint-Donat, On Petri's analysis of the linear system of quadrics through a canonical curve, Math. Ann., 206 (1973), 157-175. MR0337983 (49:2752)

[Sch1] F. O. Schreyer, Syzygies of canonical curves and special linear series, Math. Ann., 275 (1986), 105-137. MR849058 (87j:14052)

[Sch2] F. O. Schreyer, A standard basis approach to syzygies of canonical curves, J. Reine Angew. Math., 421 (1991), 83-123. MR1129577 (92j:14040)

[Za] O. Zariski, A simplified proof for the resolution of singularities of an algebraic surface, Ann. of Math. (2) 43 (1942), 583-593. MR0006851 (4:52c)

Dipartimento di Matematica, Politecnico di Torino, C.so Duca degli Abruzzi 24, 10129 TORINo, ItAaly

E-mail address: casnati@calvino.polito.it 